\title{
Special issue on physical virology
}

\author{
Françoise Livolant • Vladimir Lorman • Matteo Marsili • \\ Cristian Micheletti $\cdot$ Rudolf Podgornik
}

Published online: 3 May 2013

(C) Springer Science+Business Media Dordrecht 2013

Compared to organisms such as bacteria and eukaryotic cells, viruses have such a low level of complexity that they were at first regarded as being proto-organisms, at the borderline between living and inanimate matter. This view was gradually superseded by the recognition that viruses are highly efficient organisms which have evolved in order to hijack the "active" biological machinery of the infected cells.

Recent studies revealed how physical mechanisms are used by the viruses for guiding all salient steps of their "life cycle" This fact has also strongly motivated the recent upsurge of theoretical and experimental studies addressing the molecular basis of the key problems in virology. Specifically, the topics that are presently investigated most intensively include the following: the self-assembly and maturation of viral capsids, the functioning of the

F. Livolant · V. Lorman

Laboratoire de Physique des Solides, CNRS UMR 8502, Bat 510,

Universite Paris Sud-XI, 91405 Orsay Cedex, France

V. Lorman

Laboratoire Charles Coulomb, CNRS UMR 5221, Université Montpellier II,

Pl. E. Bataillon, 34095 Montpellier, France

M. Marsili

The Abdus Salam International Centre for Theoretical Physics,

Strada Costiera 11, 34014 Trieste, Italy

C. Micheletti

SISSA, Via Bonomea 265, 34136 Trieste, Italy

R. Podgornik ( $\varangle)$

University of Ljubljana, Ljubljana, Slovenia

e-mail: rudipod@gmail.com

R. Podgornik

IJS, Jamova 39, 1000 Ljubljana, Slovenia

R. Podgornik

University of Massachusetts, Amherst, MA, USA 
molecular motors that loads the viral genome inside preformed capsids, the conformational arrangement of the DNA or RNA inside capsids, the physical forces at play during the ejection of the viral DNA into the host cell, and the electrostatic interactions between the nucleic acids and coat proteins and virus-based nano-composites. The abovementioned efforts in characterizing the key molecular aspects of single viral particles are complemented by the very active research in the modeling of viral epidemics where, again, physics-based statistical mechanical approaches are widely used.

A "physical virology" topical conference held at the ICTP in 2012 provided a timely state-of-the art perspective on the subject that is rapidly progressing because of the ongoing advancements in experimental techniques (cryo-EM imaging and single-molecule manipulation) and theoretical ones (large-scale simulations). It came at a very timely junction as the first Gordon conference on physical virology was held in 2009 in Galveston, Texas, and the latest one in Ventura, California, in 2013, which could be taken as the defining events in the establishment of the whole field of physical virology.

This special issue of the Journal of Biological Physics is entirely dedicated to physical virology and aims to present a broad overview of the current state of the art in various subtopics of physical virology and stimulate additional activity in this rapidly developing field. 